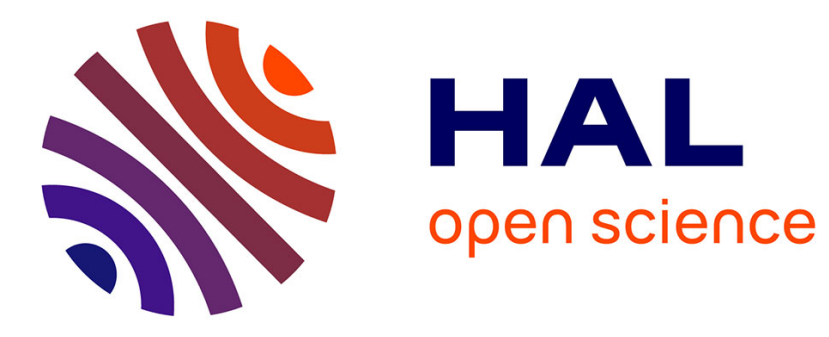

\title{
Simulation of a confined turbulent round jet at moderate Reynolds number
}

Georges Halim Atallah, Emmanuel Belut, Sullivan Lechêne, Benoît Trouette, Stéphane Vincent

\section{- To cite this version:}

Georges Halim Atallah, Emmanuel Belut, Sullivan Lechêne, Benoît Trouette, Stéphane Vincent. Simulation of a confined turbulent round jet at moderate Reynolds number. Turbulence and Interactions, 2021, 10.1007/978-3-030-65820-5_10 . hal-03263176

\section{HAL Id: hal-03263176 https://hal.science/hal-03263176}

Submitted on 17 Jun 2021

HAL is a multi-disciplinary open access archive for the deposit and dissemination of scientific research documents, whether they are published or not. The documents may come from teaching and research institutions in France or abroad, or from public or private research centers.
L'archive ouverte pluridisciplinaire HAL, est destinée au dépôt et à la diffusion de documents scientifiques de niveau recherche, publiés ou non, émanant des établissements d'enseignement et de recherche français ou étrangers, des laboratoires publics ou privés. 


\title{
Simulation of a confined turbulent round jet at moderate Reynolds number
}

\author{
Georges Halim Atallah ${ }^{1,2}$, Emmanuel Belut ${ }^{1}$, Sullivan Lechêne ${ }^{1}$, \\ Benoît Trouette ${ }^{2}$, and Stéphane Vincent ${ }^{2}$ \\ 1 INRS, French National Research and Safety Institute for the Prevention of \\ Occupational Accidents and Diseases, Lab. Aeraulic engineering, Vandœuvre, France \\ ${ }^{2}$ Université Paris-Est, Laboratoire Modélisation et Simulation Multi Echelle, \\ MSME UMR 8208, CNRS, UPEC, UPEM, F-77454, Marne-la-Vallée, France \\ georges.halimatallah@\{inrs.fr,u-pem.fr\}
}

\begin{abstract}
The objective of this study is to numerically simulate the turbulent flow of a confined round jet at moderate Reynolds number, which is representative, in a first step, of an available experiment characterizing a problem of pollutant transport in a confined medium.
\end{abstract}

Keywords: Confined round jet · Moderate Reynolds number · comparison between DNS and experiments · LES

\section{Introduction}

In numerous scientific research areas, particularly in chemistry, operators are very often required to handle materials that could be volatile and could probably be inhaled. Depending on the materials being studied, the gases generated can be toxic and therefore present a real danger to the operator's health in case of inhalation. Laboratory fume cupboards are devices that are supposed to ensure the protection of the operator. Their ability to contain pollutant gases is vulnerable to turbulent phenomena and aeraulic perturbations, which are themselves induced by drafts or moving objects. When a laboratory fume cupboard is disturbed, the pollutant gas, instead of being confined inside the device, leaks because of the induced turbulent flow. In order to simulate the pollutant transport process when the device is submitted to disturbances, we first propose to design a small-scale experimental model involving the same physical phenomena. This is an experiment describing the transport of pollutant injected into a confined enclosure through a round turbulent jet that allows to collect experimental data of time average velocity, kinetic energy and mean age air profile $[2,4]$ so that numerical simulations can be validated against experimental measurements and statistics. In the present work, we only focus on flow characteristics and more particularly on the turbulent aspect of the carrier flow motion. 


\section{Confined turbulent round jet at moderate Reynolds number}

\section{$2.1 \quad$ Experimental setup}

Air is injected into a rectangular enclosure containing the same fluid at rest through a circular inlet (round jet) at a flow rate of $40 \mathrm{~L} \cdot \mathrm{min}^{-1}$ and exits through an open circular outlet at atmospheric pressure. The diameter of the inlet injector is $D=0.04 \mathrm{~m}$, so that the Reynolds number based on the injector diameter $\operatorname{Re}=1500$. The flow rate is sufficiently high so that the flow degenerates into turbulence [1]. Airflow velocity profiles were measured by Laser Doppler Anemometry in the central plane $(y=0 \mathrm{~m})$ in several cross-sections located at $x=0.1,0.3,0.5$ and $0.7 \mathrm{~m}$ in the streamwise $x$-direction [2].

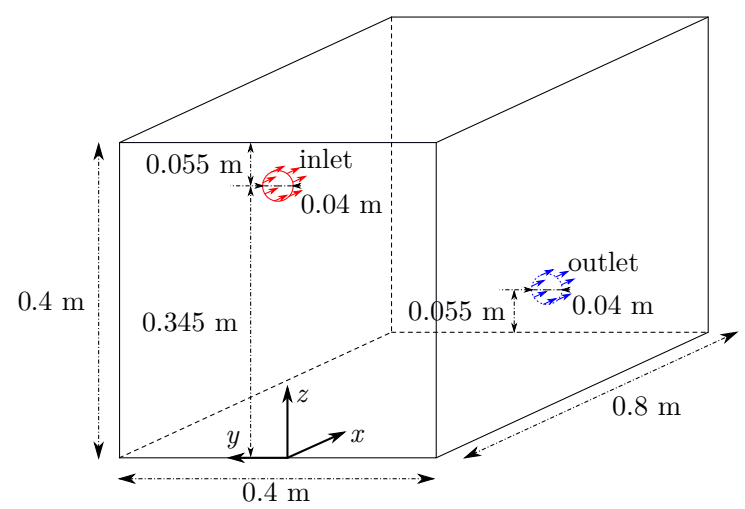

Fig. 1. Geometric description of the confined air injection in a cavity with cylindridal inlet and outlet.

\subsection{Model and numerical methods}

All developments are investigated with the home made code Fugu, developped in the TCM team of MSME lab. We performed numerical simulation of an airflow assumed to be incompressible and isothermal using a finite volume method on an irregular and staggered Cartesian grid governed by the following set of equations:

$$
\begin{aligned}
& \boldsymbol{\nabla} \cdot \boldsymbol{u}=0 \\
& \rho\left(\frac{\partial \boldsymbol{u}}{\partial t}+\nabla \cdot(\boldsymbol{u} \otimes \boldsymbol{u})\right)=-\nabla p+\nabla \cdot\left[\left(\mu+\mu_{\mathrm{sgs}}\right)\left(\boldsymbol{\nabla u}+(\boldsymbol{\nabla} \boldsymbol{u})^{T}\right)\right],
\end{aligned}
$$

where $\boldsymbol{u}$ is the velocity vector, $p$ is the pressure, $\rho=1.2250 \mathrm{~kg} \cdot \mathrm{m}^{-3}$ is the density and $\mu=1.7894 \cdot 10^{-5} \mathrm{~Pa} \cdot \mathrm{s}$ is the dynamic viscosity of the fluid. When a 
turbulence model is considered, a turbulent viscosity $\mu_{\mathrm{sgs}}$ is incorporated through an eddy viscosity model in the motion equations. In the present work, gravity effects are neglected. Boundary conditions for $\boldsymbol{u}$ are no-slip condition for all walls and zero gradient at the outlet for the normal component. Imposing a velocity profile such as the one of the experience requires taking into account a number of factors that are difficult to simulate. In practice, the injector nozzle is never really designed with perfect geometry and therefore has imperfections. They can then cause instabilities that directly affect the potential core area (nearfield) of the jet. Thus, a simple flat velocity profile such that $u^{\text {inlet }}=\mu \operatorname{Re} / \rho D$ is imposed at the inlet and only intermediate and far fields region of the jet are investigated and compared to experiments in the present work. A centered scheme is used to discretize the advection term and viscous terms whereas time integration is carried out with a second order Gear scheme. Pressure is obtained by using a time-splitting approach for handling pressure-velocity coupling. A scalar projection method is considered here [3]. These schemes are combined with the preconditioned MILU-BICGSTAB II solver to build a solution of equations (1a-1b).

A mesh convergence study was first performed with direct numerical simulation (DNS) to assess if the obtained numerical solutions reproduce the behavior of experimental data. The mesh convergence study was performed with mesh sizes of $\left\{n_{x}, n_{y}, n_{z}\right\}=\{128,64,64\},\{192,96,96\}$ and $\{256,128,128\}$. For each case, the mesh is refined with 400, 900 and 1600 cells respectively along the jet inlet cross section. It was observed that a simple regular mesh size generates too much dissipation for the same number of cells, which inhibits the ability to capture a degeneration to turbulence. This is why we chose to use a refined irregular mesh in the jet inlet cross section to capture the effects of turbulence. The time step is defined as $\Delta t=\mathrm{CFL} \times h_{\min }$ with $\mathrm{CFL}=0.5$ and $h_{\text {min }}$ is the length of the smallest cell. The flow was also simulated with Large Eddy Simulation (LES) models on the coarsest mesh size. The idea is to measure the capability of LES to provide a suitable solution on a too coarse mesh for tackling with DNS.

\subsection{Large Eddy Simulation (LES) turbulence modeling}

Our LES simulations were performed with a variety of classical LES models [6]. The first is an eddy viscosity model based on the mixed scale approach, which is a combination of the Smagorinsky and the Turbulent Kinetic Energy (TKE) models. The subgrid-scale viscosity is classically evaluated by the following formula:

$$
\nu_{\mathrm{sgs}}=C_{\mathrm{m}} \bar{\Delta}^{1+\alpha}\left(2 \bar{S}_{i j} \bar{S}_{i j}\right)^{\alpha / 2}\left(q_{c}^{2}\right)^{(1-\alpha) / 2}
$$

with $C_{\mathrm{m}}=C_{\mathrm{s}}^{2 \alpha} C_{\mathrm{TKE}}^{1-\alpha}, \bar{\Delta}=(\Delta x \Delta y \Delta z)^{1 / 3}, \bar{S}_{i j}$ is the resolved strain rate tensor, $q_{c}$ is the subgrid-scale kinetic energy. In our implementation, $\alpha \in[0,1]$ is a weighting coefficient ( $\alpha=0$ gives the TKE model, $\alpha=1$ gives the Smagorinsky model). In our approach, $\alpha$ is taken equal to 0.5 for the mixed scale model. $C_{\mathrm{s}}$ and $C_{\mathrm{TKE}}$ are the constant of the Smagorinsky and TKE models and are taken 
equal to 0.18 and 0.2 respectively. In the motion equations, $\mu_{\mathrm{sgs}}=\rho \nu_{\mathrm{sgs}}$ is then added to the molecular dynamic viscosity as shown in equation (1b).

The other one is the Wall Adaptating Local Eddy-viscosity (WALE) model [6]. Its known advantage is that it allows to reproduce a good asymptotic behaviour near solid walls for wall bounded flows. The subgrid-scale viscosity is define as:

$$
\nu_{\mathrm{sgs}}=\left(C_{w} \bar{\Delta}\right)^{2} \frac{\left(\bar{S}_{i j}^{d} \bar{S}_{i j}^{d}\right)^{3 / 2}}{\left(\bar{S}_{i j} \bar{S}_{i j}\right)^{5 / 2}+\left(\bar{S}_{i j}^{d} \bar{S}_{i j}^{d}\right)^{5 / 4}},
$$

with $C_{w}=0.75$ and $\bar{S}_{i j}^{d}=\bar{S}_{i k} \bar{S}_{k j}+\bar{\Omega}_{i k} \bar{\Omega}_{k j}-\frac{1}{3}\left(\bar{S}_{m n} \bar{S}_{m n}-\bar{\Omega}_{m n} \bar{\Omega}_{m n}\right) \delta_{i j}$. $\bar{\Omega}_{i j}$ is the resolved rotation tensor and $\delta_{i j}$ is the Kronecker delta.

\section{Results and discussion}

Direct Numerical Simulation When possible, DNS is the simplest model for turbulence and also the more accurate to investigate on a numerical point of view. Its main drawback is the requirement of solving all the time and space scales of the flow, which is generally impossible as soon as the Reynolds number is high. In our experiment of turbulent round jet, the Reynolds number is moderate, so it is reasonable to try to simulate the problem with DNS. If we succeed, a reference simulation will be available, which could be degenerated in terms of mesh or time step in order to try to simulate faster the same problem with LES models.

After thirty seconds of physical time corresponding to an established jet motion, average fields are then calculated over thirty seconds of physical time, ensuting a converged behavior of these average fields. As shown in Fig. 2, outside the jet area $(z \leq 0.3 \mathrm{~m})$, we can observe a little influence of the mesh size. In these zones, the flow is mainly laminar and all grids capture the correct motion. Inside the jet $(z>0.3 \mathrm{~m})$, numerical solutions on all meshes globally reproduces the average velocity intensities. However, refining the grids allows to converge simulation results to those of the experiments, which is a nice feature of the Fugu code. Note that the fact that the maximum velocities are not at the same height $z$ is explained by the fact that the imposed velocity profile is spatially constant in the injector, which is not the case in the experiment [2].

The DNS gives results in fairly good agreement with those of the experiments, but the computation cost is far too high on the finer mesh, even with the MPI parallel implementation that we considered (512 processors). An important point is to be able to carry out this kind of simulation with mesh sizes of the order of the coarsest grid presented here $(128 \times 64 \times 64$ cells $)$. Therefore, in the next section, LES simulations with models presented in Sect. 2.3 were performed on the coarsest mesh. 

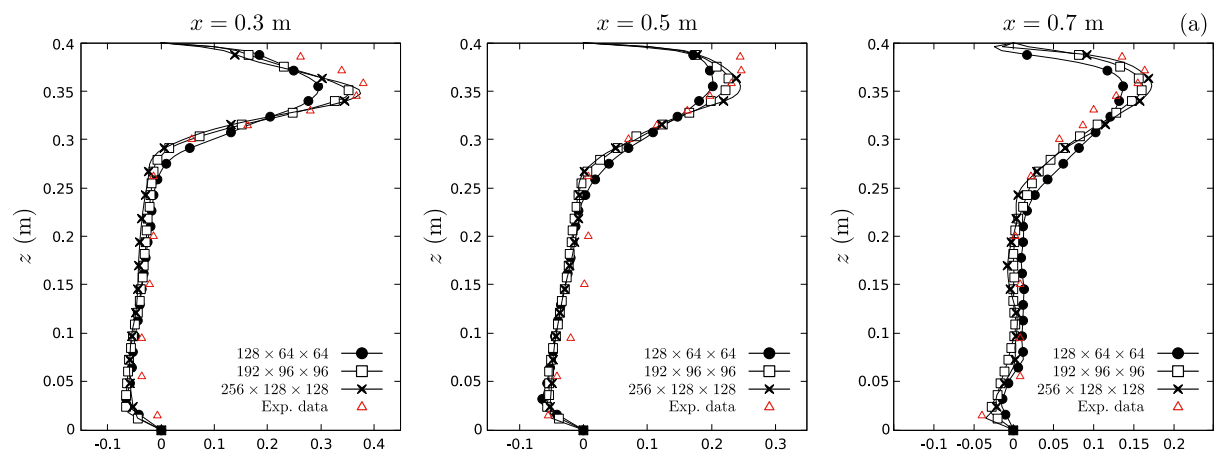

$\langle u\rangle(\mathrm{m} / \mathrm{s})$

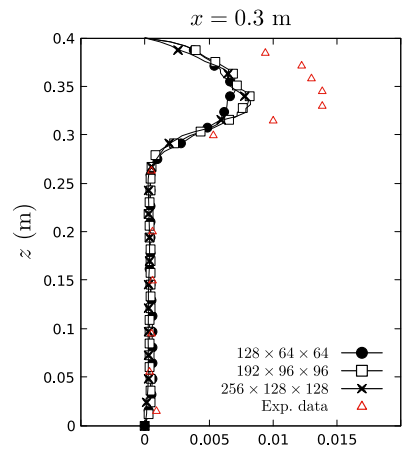

$\langle u\rangle(\mathrm{m} / \mathrm{s})$

$\langle u\rangle(\mathrm{m} / \mathrm{s})$
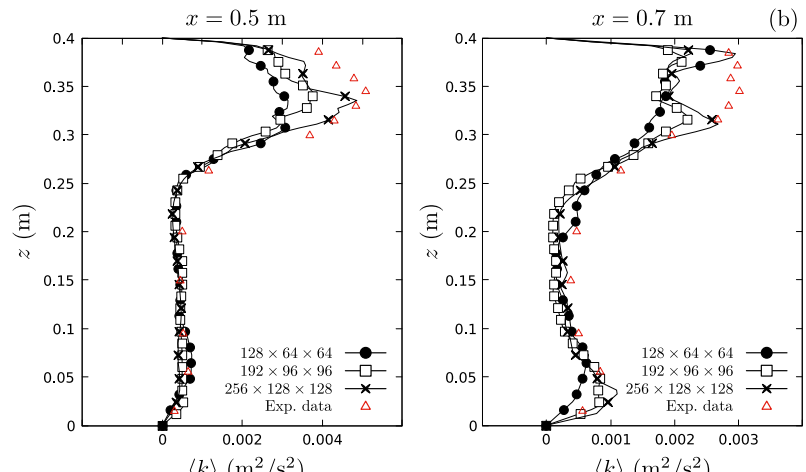

$\langle k\rangle\left(\mathrm{m}^{2} / \mathrm{s}^{2}\right)$

$\langle k\rangle\left(\mathrm{m}^{2} / \mathrm{s}^{2}\right)$

$\langle k\rangle\left(\mathrm{m}^{2} / \mathrm{s}^{2}\right)$

Fig. 2. Measured and computed (a) mean velocity profiles and (b) mean turbulent kinetic energy at the central plane $y=0 \mathrm{~m}$ and $x=0.3,0.5$ and $0.7 \mathrm{~m}$. Comparison between DNS simulations (without explicit LES model) and experiments [2].

Large Eddy Simulation In order to reach such results as in DNS on fine mesh at a lower cost, simulations were performed on the coarsest mesh using LES models with the same numerical setup as in DNS. A first observation is that the three Smagorinsky, TKE and mixed scale models maintain the jet in a laminar state and inhibit the transition to turbulence. However, the WALE model provides a better behaviour than the others despite the fact that the laminar-turbulent transition occurs farther than it occurs in DNS. This leads to an overestimation of velocity intensities as shown in Fig. 3.

\section{Concluding remarks}

The dynamics of a cylindrical jet at moderate Reynolds number of 1500 has been simulated with DNS and LES turbulence approaches. The results of the DNS compare favorably to those provided by the experiments. LES conducted on the coarsest DNS grid are not able to recover the correct experimental jet dynamics. The main reason that we have identified, by changing all physical and 


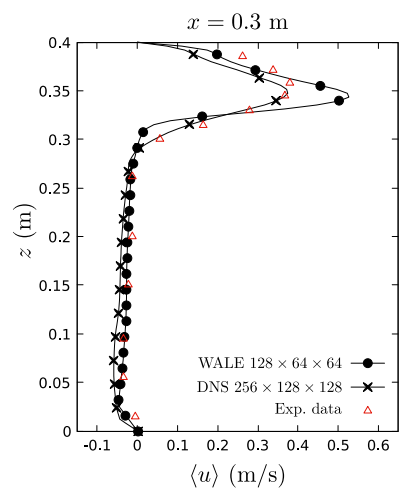

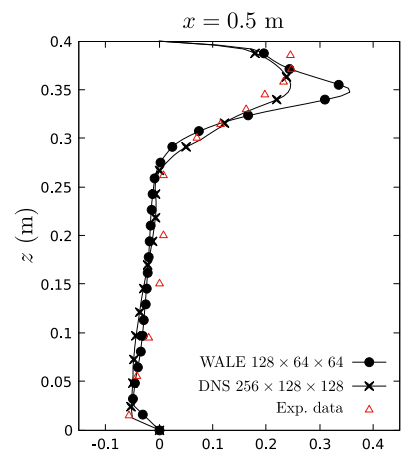

$\langle u\rangle(\mathrm{m} / \mathrm{s})$

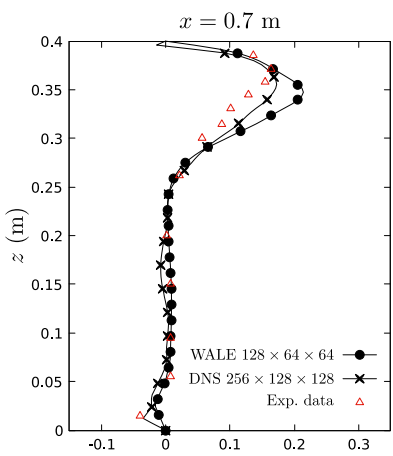

$\langle u\rangle(\mathrm{m} / \mathrm{s})$

Fig. 3. Comparison between computed mean velocity at the central plane $y=0 \mathrm{~m}$ and $x=0.3,0.5$ and $0.7 \mathrm{~m}$ with LES WALE model and DNS on $256 \times 128 \times 128$ mesh size.

numerical parameters of the simulations, is that the results are very sensitive to inlet jet profile of mean velocity (for the DNS) and also to the fluctuating (or subgrid component) of the velocity, that is not considered in the present work. Future improvements of our LES simulations will be to impose synthetic turbulent unsteady inlet jet conditions [5] in order to feed simulations with realistic subgrid scale fluctuations of the velocity.

\section{Acknowledgements}

The authors are grateful for access to the computational facilities of the French CINES (National computing center for higher education) and CCRT (National computing center of CEA) under project number A0032B06115.

\section{References}

1. Abdel-Rahman, A.: A review of effects of initial and boundary conditions on turbulent jets. WSEAS transactions on Fluid Mechanics 4(5), 257-275 (2010)

2. Belut, E., Christophe, T.: A new experimental dataset to validate cfd models of airborne nanoparticles agglomeration (05 2016)

3. Goda, K.: A multistep technique with implicit difference schemes for calculating two- or three-dimensional cavity flows. J. Comput. Phys. 30(1), 76 - 95 (1979)

4. Guichard, R., Belut, E.: Simulation of airborne nanoparticles transport, deposition and aggregation: Experimental validation of a cfd-qmom approach. Journal of Aerosol Science 104, 16 - 31 (2017)

5. Klein, M., Sadiki, A., Janicka, J.: A digital filter based generation of inflow data for spatially developing direct numerical or large eddy simulations. Journal of Computational Physics 186(2), 652 - 665 (2003)

6. Sagaut, P., Meneveau, C.: Large Eddy Simulation for Incompressible Flows: An Introduction. Scientific Computation, Springer (2006) 